\title{
Macroscopic Quantum Tunneling of a Bose Condensate
}

\author{
H.T.C. Stoof \\ University of Utrecht, Institute for Theoretical Physics, Princetonplein 5, \\ P.O. Box 80.006, 3508 TA Utrecht, The Netherlands
}

\begin{abstract}
We study, by means of a variational method, the stability of a condensate in a magnetically trapped atomic Bose gas with a negative scattering length and find that the condensate is unstable in general. However, for temperatures sufficiently close to the critical temperature the condensate turns out to be metastable. For that case we determine in the usual WKB approximation the decay rate of the condensate due to macroscopic quantum fluctuations. When appropriate, we also calculate the decay rate due to thermal fluctuations. An important feature of our approach is that (nonsingular) phase fluctuations of the condensate are taken into account exactly.
\end{abstract}

PACS numbers: $67.40 .-\mathrm{w}, 32.80 . \mathrm{Pj}, 42.50 . \mathrm{Vk}$ 


\section{INTRODUCTION}

The observation of Bose-Einstein condensation in dilute atomic ${ }^{87} \mathrm{Rb}$ [1], ${ }^{7} \mathrm{Li}$ [2], and ${ }^{23} \mathrm{Na}$ [3] vapors last year, has created a great deal of excitement in the atomic physics community. Although it was speculated upon already for some time, the actual observation of the condensation phenomenon in these alkali gases nevertheless came as somewhat of a surprise, because in the spring of 1995 experiments with a trapped atomic hydrogen gas still held the record in achieving the necessary conditions for Bose-Einstein condensation [4, 5]. The main reasons for this unexpected turn of events appear to be that alkali atoms are much more easy to detect and that experiments with alkali vapors can be performed at room temperature in contrast with the cryogenic environment that is required for experiments with an atomic hydrogen gas.

The report of Bose-Einstein condensation in atomic ${ }^{7} \mathrm{Li}$ was also surprising for another, more fundamental, reason. It had namely been established experimentally [6] that the effective interaction between two ${ }^{7} \mathrm{Li}$ atoms is attractive, or more precisely that the s-wave scattering length $a$ is negative. For a homogeneous gas this implies that Bose-Einstein condensation cannot take place in the mechanically (meta)stable region of the phase diagram and is preempted by a first-order gas-liquid or gas-solid transition [7]. Although first Hulet (8) and subsequently also Ruprecht et al. 90 had suggested that this conclusion holds in an inhomogeneous situation only if the number of atoms is sufficiently large, the number of atoms used in the experiment indeed appeared to be too large by about two orders of magnitude.

At present, this unsatisfactory state of affairs still exists, i.e. experiment claims to observe a condensate in a gas with a negative scattering length whereas theory seems to predict that this should not be possible. In an attempt to bridge at least part of the gap between theory and experiment, we study in Sec. III for zero and subsequently also for nonzero temperatures, the stability of a condensate of ${ }^{7} \mathrm{Li}$ atoms in an isotropic harmonic oscillator potential. In the process of this analysis, we show that there exists an interesting analogy between the 
quantum dynamics of the condensate and the quantum mechanics of a particle in an unstable potential. Therefore, to make the paper more selfcontained and to bring out this analogy most clearly, we present first in Sec. [I] a brief summary of how the WKB approximation to the tunneling rate of a particle is derived by path-integral methods. We end in Sec. $\mathbb{\square}$ with some conclusions.

\section{TUNNELING OF A PARTICLE}

The tunneling rate $\Gamma_{0}$ of a particle with mass $m^{*}$ out of a metastable minimum of a potential $V(q)$ can be calculated by means of the relation

$$
\Gamma_{0}=-\frac{2}{\hbar} \operatorname{Im}\left(E_{0}\right)=\lim _{T \downarrow 0} \frac{2 k_{B} T}{\hbar} \operatorname{Im}(\ln Z)
$$

where $\operatorname{Im}\left(E_{0}\right)$ is the imaginary part of the (analytically continued) groundstate energy in the metastable minimum of the potential, and $Z=\operatorname{Tr}\left[e^{-\beta H}\right]$ is the partition function with $\beta=1 / k_{B} T$ and $H(p, q)=p^{2} / 2 m^{*}+V(q)$ the usual Hamilton operator for the particle. This is a convenient starting point for our discussion, because the partition function can in the usual way be represented as a functional integral over the functions $q(\tau)$ and $p(\tau)$, i.e.

$$
Z=\int d[q] \int d[p] \exp \left\{-\frac{1}{\hbar} \int_{-\hbar \beta / 2}^{\hbar \beta / 2} d \tau\left(-i p \frac{d q}{d \tau}+H(p, q)\right)\right\}
$$

with the periodic boundary condition $q(-\hbar \beta / 2)=q(\hbar \beta / 2)$ on the coordinate but no restrictions on the momentum. For the hamiltonian of interest the integral over the momentum $p(\tau)$ is just a gaussian that can be easily carried out. As a result the partition function is in this case also equal to a path integral $\int d[q] \exp \{-S[q] / \hbar\}$ over all periodic paths $q(\tau)$ and with a (Euclidian) action given by

$$
S[q]=\int_{-\hbar \beta / 2}^{\hbar \beta / 2} d \tau\left(\frac{1}{2} m^{*}\left(\frac{d q}{d \tau}\right)^{2}+V(q)\right) .
$$

Our task is therefore to evaluate this path integral. In general this cannot be done exactly, but one can obtain a good (semiclassical) approximation by noting that the dominant 
contributions to the path integral are from paths that minimize the action $S[q]$. Such paths are solutions to the Euler-Lagrange equation

$$
m^{*} \frac{d^{2} q}{d \tau^{2}}=-\frac{d(-V(q))}{d q}
$$

which has the same form as the classical equation of motion for a particle with mass $m^{*}$ in a potential $-V(q)$. A periodic solution to this equation is therefore $q(\tau)=q_{0}$, where $q_{0}$ is the position of the metastable minimum and obeys $d V\left(q_{0}\right) / d q=0$. Writing $q(\tau)=q_{0}+q^{\prime}(\tau)$ and expanding the action up to quadratic order in the fluctuations $q^{\prime}(\tau)$, we obtain first of all that

$$
Z \simeq e^{-\beta V\left(q_{0}\right)} \int d\left[q^{\prime}\right] \exp \left\{-\frac{1}{\hbar} \int_{-\hbar \beta / 2}^{\hbar \beta / 2} d \tau\left(\frac{1}{2} m^{*}\left(\frac{d q^{\prime}}{d \tau}\right)^{2}+\frac{1}{2} \frac{d^{2} V\left(q_{0}\right)}{d q^{2}} q^{\prime 2}\right)\right\}
$$

Introducing $m^{*} \omega_{0}^{2} \equiv d^{2} V\left(q_{0}\right) / d q^{2}$, we notice that the path integral in the right-hand side is just equal to the partition function for a harmonic oscillator and therefore that

$$
\begin{aligned}
& \int d\left[q^{\prime}\right] \exp \left\{-\frac{1}{2} \int_{-\hbar \beta / 2}^{\hbar \beta / 2} d \tau q^{\prime}\left(-\frac{m^{*}}{\hbar} \frac{d^{2}}{d \tau^{2}}+\frac{m^{*} \omega_{0}^{2}}{\hbar}\right) q^{\prime}\right\} \\
& =\mathcal{N}\left\{\operatorname{det}\left[\frac{m^{*}}{2 \pi \hbar}\left(-\frac{d^{2}}{d \tau^{2}}+\omega_{0}^{2}\right)\right]\right\}^{-1 / 2}=\exp \left\{-\beta \frac{\hbar \omega_{0}}{2}-\ln \left(1-e^{-\beta \hbar \omega_{0}}\right)\right\},
\end{aligned}
$$

denoting the usual normalization factor due to the measure in the path integral by $\mathcal{N}$. Taking now the limit $T \downarrow 0$ (or $\beta \rightarrow \infty$ ) we thus find in this approximation for the groundstate energy $E_{0} \simeq V\left(q_{0}\right)+\hbar \omega_{0} / 2$ and for the tunneling rate $\Gamma_{0}=0$. This is clearly a reasonable first-order result that is due to the fact that we can in first instance always approximate the full potential $V(q)$ by the harmonic oscillator potential $V\left(q_{0}\right)+m^{*} \omega_{0}^{2}\left(q-q_{0}\right)^{2} / 2$.

To obtain a nonzero value for the tunneling rate we must realize that there is another periodic solution to Eq. (4) that has an action which (in the zero temperature limit) is only slightly different from $S\left[q_{0}\right]$ and therefore also gives an important contribution to the partition function. Using the classical analogy, this so-called 'bounce' solution $q_{b}(\tau)$ has the property that the particle spends a very long time around $q_{0}$ but in a relatively short time oscillates once in the potential minimum of $-V(q)$, i.e. it bounces from $q_{0}$ to $q_{1}$ and 
back to $q_{0}$, where $q_{1}$ obeys $V\left(q_{1}\right)=V\left(q_{0}\right)$. In particular, for $\tau \rightarrow \pm \infty$ it behaves as $q_{b}(\tau) \sim q_{0} \mp\left(v_{0} / \omega_{0}\right) e^{-\omega_{0}|\tau|}$ with $v_{0}$ determined by the details of the potential.

Proceeding as before by writing $q(\tau)=q_{b}(\tau)+q^{\prime}(\tau)$ and expanding the action up to quadratic order in $q^{\prime}(\tau)$, we now find that near zero temperature the partition function equals

$$
Z \simeq e^{\beta\left(V\left(q_{0}\right)+\hbar \omega_{0} / 2\right)}\left(1+\left[\frac{\operatorname{det}\left(-d^{2} / d \tau^{2}+\omega_{0}^{2}\right)}{\operatorname{det}\left(-d^{2} / d \tau^{2}+\omega_{b}(\tau)^{2}\right)}\right]^{1 / 2} e^{-\left(S\left[q_{b}\right]-S\left[q_{0}\right]\right) / \hbar}\right)
$$

introducing the quantity $\omega_{b}(\tau)$ by means of $m^{*} \omega_{b}(\tau)^{2} \equiv d^{2} V\left(q_{b}(\tau)\right) / d q^{2}$. Adding in a similar manner also the contributions from paths with an arbitrary number of bounces, we find that the series exponentiates and hence that

$$
E_{0} \simeq V\left(q_{0}\right)+\frac{\hbar \omega_{0}}{2}-\lim _{T \downarrow 0} k_{B} T\left[\frac{\operatorname{det}\left(-d^{2} / d \tau^{2}+\omega_{0}^{2}\right)}{\operatorname{det}\left(-d^{2} / d \tau^{2}+\omega_{b}(\tau)^{2}\right)}\right]^{1 / 2} e^{-\left(S\left[q_{b}\right]-S\left[q_{0}\right]\right) / \hbar}
$$

We expect the third term in the right-hand side to represent the tunneling rate out of the metastable minimum and therefore to be purely imaginary. This expectation is indeed correct, because the operator $-d^{2} / d \tau^{2}+\omega_{b}(\tau)^{2}$ turns out to have a negative eigenvalue. This is most easily understood from the fact that Eq. (4) shows that $d q_{b}(\tau) / d \tau$ is an eigenfunction of this operator with an eigenvalue equal to zero. Since this eigenfunction has one node, we know from our experience with the Schrödinger equation that there must be an eigenfunction without nodes that has a lower, and therefore, negative eigenvalue.

However, the presence of an eigenvalue equal to zero appears to give an infinite result for the ratio of determinants in Eq. (8). Fortunately, this is due to an improper treatment of the zero mode in the calculation of the path integral over the fluctuations $q^{\prime}(\tau)$. Since $q_{b}\left(\tau-\tau_{0}\right)=q_{b}(\tau)-\tau_{0} d q_{b}(\tau) / d \tau+O\left(\tau_{0}^{2}\right)$, we note that this zero mode is just associated with a translation of the 'bounce' solution $q_{b}(\tau)$ and hence that the square root of the ratio of determinants must be proportional to the total time interval $\hbar \beta=\hbar / k_{B} T$. A detailed and beautiful analysis by Duru et al. actually shows that

$$
\lim _{T \downarrow 0} k_{B} T\left[\frac{\operatorname{det}\left(-d^{2} / d \tau^{2}+\omega_{0}^{2}\right)}{\operatorname{det}\left(-d^{2} / d \tau^{2}+\omega_{b}(\tau)^{2}\right)}\right]^{1 / 2}=\frac{i}{2} \sqrt{\frac{m^{*} v_{0}^{2} \hbar \omega_{0}}{\pi}} .
$$


Combining this with Eq. (8) we finally arrive at

$$
\Gamma_{0}=-\frac{2}{\hbar} \operatorname{Im}\left(E_{0}\right)=\sqrt{\frac{m^{*} \omega_{0} v_{0}^{2}}{\pi \hbar}} e^{-\left(S\left[q_{b}\right]-S\left[q_{0}\right]\right) / \hbar}
$$

where

$$
\frac{S\left[q_{b}\right]-S\left[q_{0}\right]}{\hbar}=\frac{2}{\hbar} \int_{q_{0}}^{q_{1}} d q \sqrt{2 m^{*}\left(V(q)-V\left(q_{0}\right)\right)}
$$

is recognized as the usual WKB expression for the exponent of the tunneling rate.

\section{TUNNELING OF A CONDENSATE}

We now turn to the problem of the stability of the condensate in a magnetically trapped gas of ${ }^{7} \mathrm{Li}$ atoms. We consider here only the experimentally relevant case of a large number of particles $N \gg 1$ in a large trap. Quantitatively, the latter means that $r_{V} / \ell \ll 1$, where $r_{V}$ is the range of the interatomic interaction, $\ell=\sqrt{2 \pi \hbar / m \omega}$ is the spatial extent of the one-particle ground state in a harmonic oscillator potential with level spacing $\hbar \omega$, and $m$ is the mass of the ${ }^{7} \mathrm{Li}$ atoms. (In the case of ${ }^{7} \mathrm{Li}$ the above condition also implies that $|a| / \ell \ll 1$.) Moreover, we will always require that the density $n$ in the center in the trap is such that the gas parameter $n r_{V}^{3} \ll 1$. This basically leads to an upper bound on the total number of particles that is always satisfied by the experiment of interest [2].

\section{A. The case $\mathbf{T}=0$}

At zero temperature and in an external trapping potential $V^{e x t}(\vec{x})=m \omega^{2} \vec{x}^{2} / 2$, the effective hamiltonian for the condensate wavefunction $\psi(\vec{x}, t)$ and its canonical momentum $\pi(\vec{x}, t)=i \hbar \psi^{*}(\vec{x}, t)$ is given by

$$
H[\pi, \psi]=\int d \vec{x} \psi^{*}\left(-\frac{\hbar^{2}}{2 m} \nabla^{2}+V^{e x t}+\frac{T^{2 B}(\overrightarrow{0}, \overrightarrow{0} ; 0)}{2}|\psi|^{2}\right) \psi
$$

since then the Hamilton equations exactly reproduce the nonlinear Schrödinger equations for $\psi(\vec{x}, t)$ and $\psi^{*}(\vec{x}, t)$, respectively. For example, we correctly find that 


$$
\begin{aligned}
\frac{\partial}{\partial t} \psi(\vec{x}, t) & \equiv \frac{\delta}{\delta \pi(\vec{x}, t)} H[\pi, \psi] \\
& =\frac{1}{i \hbar}\left(-\frac{\hbar^{2}}{2 m} \nabla^{2}+V^{e x t}(\vec{x})+T^{2 B}(\overrightarrow{0}, \overrightarrow{0} ; 0)|\psi(\vec{x}, t)|^{2}\right) \psi(\vec{x}, t)
\end{aligned}
$$

with $T^{2 B}(\overrightarrow{0}, \overrightarrow{0} ; 0)=4 \pi a \hbar^{2} / m$ the effective interaction between the atoms. It is important to mention here that, due to infrared divergences in the theory of the dilute Bose gas [11, this nonlinear Schrödinger equation is only valid if the energy cut-off provided by the trapping potential is sufficiently large. More precisely we must require that $\hbar \omega>4 \pi n|a| \hbar^{2} / m$. Fortunately, this correponds precisely to the conditions under which the condensate is metastable as we will find out shortly.

After this brief discussion of the condensate wavefunction as a classical field, we can now turn to the quantum fluctuations of the condensate. As in Sec. II, we must then consider the partition function $Z=\operatorname{Tr}\left[e^{-\beta H}\right]$ at zero temperature. This function can be written as the functional integral

$$
Z=\int d\left[\psi^{*}\right] d[\psi] \exp \left\{-\frac{1}{\hbar} \int d \tau\left(\int d \vec{x} \psi^{*} \hbar \frac{\partial}{\partial \tau} \psi+H\left[i \hbar \psi^{*}, \psi\right]\right)\right\}
$$

over the periodic fields $\psi(\vec{x}, \tau)$ and $\psi^{*}(\vec{x}, t)$, which is the direct analog of Eq. (2). To proceed as in the case of the quantum mechanics of a particle, we should now integrate out the momentum field. This is, however, not helpful for our purposes because the hamiltonian does not have a momentum independent part. Consequently, it seems that a stability analysis of the condensate cannot be performed in the same way as in Sec. [I]

The way out of this dilemma is found by noting that the instability of the condensate is, just as in the homogeneous case, a result of density fluctuations that lead to a lower energy because of the effectively attractive interaction between the ${ }^{7} \mathrm{Li}$ atoms. It is therefore advantageous to perform a canonical transformation by means of the relation $\psi=\sqrt{\rho} e^{i \chi}$ and to use the density field $\rho(\vec{x}, \tau)$ and the phase field $\chi(\vec{x}, \tau)$ to calculate the partition function. Indeed, a simple substitution together with the periodicity of $\rho(\vec{x}, \tau)$ shows that the partition function is equal to the functional integral $\int d[\rho] \int d[\chi] \exp \{-S[\rho, \chi] / \hbar\}$ with an action 


$$
S[\rho, \chi]=\int d \tau \int d \vec{x}\left(i \hbar \rho \frac{\partial \chi}{\partial \tau}+\frac{\hbar^{2} \rho}{2 m}(\nabla \chi)^{2}+\frac{\hbar^{2}}{8 m \rho}(\nabla \rho)^{2}+V^{e x t} \rho+\frac{T^{2 B}(\overrightarrow{0}, \overrightarrow{0} ; 0)}{2} \rho^{2}\right)
$$

that is quadratic in the phase field $\chi(\vec{x}, \tau)$. Hence, we can now immediately integrate over this field.

There is an important point to be made about this integration, which reflects the fact that if the original fields $\psi(\vec{x}, \tau)$ and $\psi^{*}(\vec{x}, \tau)$ are periodic, the phase field $\chi(\vec{x}, \tau)$ is only periodic up to a multiple of $2 \pi$. To calculate the partition function correctly, we must therefore first integrate over all the fields $\chi(\vec{x}, \tau)$ obeying the boundary condition $\chi(\vec{x}, \infty)=$ $\chi(\vec{x},-\infty)+2 \pi j$ and subsequently sum over all possible integers $j$. Clearly, this change in boundary conditions affects only the zero-momentum part of $\chi(\vec{x}, \tau)$. As a result we have to consider the sum

$$
\sum_{j} \int d\left[\chi_{\overrightarrow{0}}\right] \exp \left\{-i \int d \tau N_{0} \frac{d \chi_{\overrightarrow{0}}}{d \tau}\right\}
$$

first, where we made use of the fact that the total number of particles in the condensate $N_{0}(\tau)=\int d \vec{x} \rho(\vec{x}, \tau)$ and the boundary condition $\chi_{\overrightarrow{0}}(\infty)=\chi_{\overrightarrow{0}}(-\infty)+2 \pi j$ on the integration is implicitly assumed for each term in the sum. Performing a partial integration on the integral in the exponent, this sum becomes equal to

$$
\sum_{j} e^{2 \pi i N_{0} j} \delta\left[\frac{d N_{0}}{d \tau}\right]
$$

because the integration over $\chi_{\overrightarrow{0}}(\tau)$ then simply leads to the constraint of a constant number of particles in the condensate, i.e. $N_{0}(\tau)=N_{0}$. At zero temperature and under the conditions that the nonlinear Schrödinger equation is valid, this actually implies that $N_{0}(\tau)$ is equal to the total number of particles $N$. In addition, the sum $\sum_{j} e^{2 \pi i N_{0} j}$ equals $\sum_{j} \delta\left(N_{0}-j\right)$ and thus requires that $N_{0}$ is an integer. In this manner we see explicitly that the integration over the zero-momentum part of $\rho(\vec{x}, \tau)$ is effectively only a sum over the total number of particles and, most important for our purposes, that the partition function at a constant number of particles is given by the functional integral $\int d[\rho] \int d[\chi] \exp \{-S[\rho, \chi] / \hbar\}$ over all nonzero momentum components of the density and phase fields. 
The integration over the nonzero momentum part of $\chi(\vec{x}, \tau)$ is readily accomplished by introducing the Green's function for the phase fluctuations $G\left(\vec{x}, \vec{x}^{\prime} ; \rho\right)$, that obeys

$$
\frac{\hbar}{m}\left((\nabla \rho) \cdot \nabla+\rho \nabla^{2}\right) G\left(\vec{x}, \vec{x}^{\prime} ; \rho\right)=\delta\left(\vec{x}-\vec{x}^{\prime}\right) .
$$

In terms of this Green's function the formal solution to the Euler-Lagrange equation $\delta S[\rho, \chi] / \delta \chi=0$ reads

$$
\chi(\vec{x}, \tau)=-i \int d \vec{x}^{\prime} G\left(\vec{x}, \vec{x}^{\prime} ; \rho\right) \frac{\partial \rho\left(\vec{x}^{\prime}, \tau\right)}{\partial \tau},
$$

which after a substitution in Eq. (15) gives the following effective action for the density field

$$
\begin{aligned}
S[\rho]=\int d & \int d \vec{x} \int d \vec{x}^{\prime}\left(-\frac{\hbar}{2} \frac{\partial \rho(\vec{x}, \tau)}{\partial \tau} G\left(\vec{x}, \vec{x}^{\prime} ; \rho\right) \frac{\partial \rho\left(\vec{x}^{\prime}, \tau\right)}{\partial \tau}\right) \\
& +\int d \tau \int d \vec{x}\left(\frac{\hbar^{2}}{8 m \rho}(\nabla \rho)^{2}+V^{e x t} \rho+\frac{T^{2 B}(\overrightarrow{0}, \overrightarrow{0} ; 0)}{2} \rho^{2}\right) .
\end{aligned}
$$

This is the desired analog of Eq. (3) that we will now use to determine the stability of the condensate at zero temperature.

Our first task is to see whether a metastable condensate is possible at all in a trap. This question can be answered by considering only the 'potential energy'

$$
V[\rho]=\int d \vec{x}\left(\frac{\hbar^{2}}{8 m \rho}(\nabla \rho)^{2}+V^{e x t} \rho+\frac{T^{2 B}(\overrightarrow{0}, \overrightarrow{0} ; 0)}{2} \rho^{2}\right)
$$

for time-independent density configurations. Because we are unable to analytically consider all configurations, we proceed in a variational way [12] and investigate here only the gaussian profiles

$$
\rho(\vec{x} ; q)=N\left(\frac{1}{\pi q^{2}}\right)^{3 / 2} \exp \left(-\frac{\vec{x}^{2}}{q^{2}}\right) .
$$

The physical reason behind this choice is that we expect the shape of the condensate, if it is metastable, to be close to the square of the one-particle groundstate wavefunction of the trapping potential and, therefore, to be reasonably accurately described by a gaussian. We will see shortly that this expectation is indeed correct. 
Substituting the above gaussian into the expression for the 'potential energy', we find that

$$
V[\rho]=N\left(\frac{3 \hbar^{2}}{4 m q^{2}}+\frac{3}{4} m \omega^{2} q^{2}-\frac{N}{\sqrt{2 \pi}} \frac{\hbar^{2}|a|}{m q^{3}}\right) \equiv N V(q) .
$$

Hence, for $|a|=0$ the potential $V(q)$ has an absolute minimum at $q=\sqrt{\hbar / m \omega}=\ell / \sqrt{2 \pi}$ and the 'potential energy' at this minimum is $3 N \hbar \omega / 2$. Clearly, this corresponds exactly to an ideal Bose condensate. For $|a| \neq 0$ the 'potential energy' is always unbounded from below because $V(q) \rightarrow-\infty$ if $q \downarrow 0$. This implies that the condensate is in general unstable and tends to collapse to the density profile $\lim _{q \downarrow 0} \rho(\vec{x} ; q)=N \delta(\vec{x})$. Of course, in a realistic system the collapse to this density profile is ultimately prevented by the hard core of the interatomic interaction as the density reaches a value of the order of $O\left(1 / r_{V}^{3}\right)$. Although our theory certainly breaks down at these densities, the approximation $N \delta(\vec{x})$ for the final density profile is nevertheless rather accurate for the lengthscales of interest, due to the condition $r_{V} / \ell \ll 1$.

The most important feature of Eq. (21) is, however, that if the condition

$$
N \frac{|a|}{\ell}<\frac{2}{5^{5 / 4}} \simeq 0.27
$$

is fullfiled, the potential $V(q)$ has a metastable minimum. This result can directly be compared with the work of Ruprecht et al., who find by a numerical integration of the nonlinear Schrödinger equation Eq. (13) that a stable solution can only be obtained if $N|a| / \ell<0.23$ [9]. Our variational calculation, therefore, gives an upper bound that is only $16 \%$ too high. Apparently this is the amount of accuracy that one can obtain for the condensate energy by considering only the density profiles $\rho(\vec{x} ; q)$.

Having arrived at the conclusion that a metastable condensate is possible at zero temperature if the number of particles in the gas is sufficiently small, we now want to calculate the lifetime of the condensate due to macroscopic quantum fluctuations. This involves also the evaluation of the 'kinetic energy'

$$
T[\rho]=\int d \vec{x} \int d \vec{x}^{\prime}\left(-\frac{\hbar}{2} \frac{\partial \rho(\vec{x}, \tau)}{\partial \tau} G\left(\vec{x}, \vec{x}^{\prime} ; \rho\right) \frac{\partial \rho\left(\vec{x}^{\prime}, \tau\right)}{\partial \tau}\right) .
$$


Again an exact treatment of this part of the effective action is unfeasible. However, our previous discussion of the 'potential energy' suggests a reasonably accurate approximation that amounts to the promotion of the variational parameter $q$ to a real dynamical variable $q(\tau)$. Hence, we assume that for a description of the tunneling process the most important configurations of the condensate are given by $\rho(\vec{x}, \tau)=\rho(\vec{x} ; q(\tau))$. The reason for this assumption is threefold. First, we have already seen that the profile $\rho(\vec{x} ; q)$ gives an accurate description of the metastable minimum of the potential $V[\rho]$. Second, one expects that the minimum energy barrier for the tunneling process is associated with a rotationally symmetric configuration 13 that, because of the diluteness of the gas, can be represented by a gaussian with the same amount of accuracy as the metastable minimum. Third, the profile $\rho(\vec{x} ; q)$ describes in the limit $q \downarrow 0$ also the completely collapsed state of the system. In combination, the configurations $\rho(\vec{x} ; q(\tau))$ thus seem to provide a reasonable interpolation between the initial and final density profiles of the tunneling process.

Evaluating the 'kinetic energy' for the variational ansatz $\rho(\vec{x}, \tau)=\rho(\vec{x} ; q(\tau))$ and adding Eq. (21) we easily find that the quantum dynamics of the condensate is determined by the action

$$
S[q]=N \int d \tau\left(\frac{1}{2} m^{*}\left(\frac{d q}{d \tau}\right)^{2}+V(q)\right)
$$

which differs only by an overall factor $N$ from the zero temperature limit of Eq. (3). The effective mass $m^{*}$ is difficult to calculation exactly due to the inhomogeneity of the system, but can be estimated by noting that the dominant contributions to the 'kinetic energy' come from the region near the center of the trap. In this region the Green's function for the phase fluctuations is well approximated by

$$
G\left(\vec{x}, \vec{x}^{\prime} ; \rho\right) \simeq-\frac{m}{4 \pi \hbar \rho(\overrightarrow{0} ; q)} \frac{1}{\left|\vec{x}-\vec{x}^{\prime}\right|}
$$

and we obtain

$$
m^{*} \simeq \frac{m}{\pi^{5 / 2}} \int d \vec{y} \int d \vec{y}^{\prime}\left(\frac{3}{2}-\vec{y}^{2}\right) \exp \left(-\vec{y}^{2}\right) \frac{1}{\left|\vec{y}-\vec{y}^{\prime}\right|}\left(\frac{3}{2}-\vec{y}^{2}\right) \exp \left(-\vec{y}^{2}\right) \simeq 0.27 m
$$


Although the exact value of the effective mass is presumably somewhat larger than this result, it is in any case of the order of the atomic mass $m$.

Applying now the final result of Sec. II (replacing only $\hbar$ by $\hbar / N$ ) we immediately find that the decay rate of the condensate is equal to

$$
\Gamma_{0}=\sqrt{\frac{N m^{*} \omega_{0} v_{0}^{2}}{\pi \hbar}} \exp \left\{-\frac{2 N}{\hbar} \int_{q_{0}}^{q_{1}} d q \sqrt{2 m^{*}\left(V(q)-V\left(q_{0}\right)\right)}\right\}
$$

and typically very small for a number of condensate particles $N \gg 1$ but still sufficiently small to fulfill the condition of metastability. Note that both requirements do not exclude each other, because we are only considering traps for which $|a| / \ell \ll 1$.

\section{B. The case $\mathbf{T} \neq \mathbf{0}$}

We have seen that at zero temperature a metastable condensate is possible if the number of ${ }^{7} \mathrm{Li}$ atoms is less than $N_{m} \equiv 0.23 \ell /|a| \simeq 1440$. (The scattering length is $-27 a_{0}$ and the effective isotropic level splitting $\hbar\left(\omega_{x} \omega_{y} \omega_{z}\right)^{1 / 3}$ for the trap is $7 n K$ [2].) In the Rice experiment, however, one reports the observation of a condensate for temperatures as high as $400 \mathrm{nK}$ and total numbers of atoms as great as $2 \cdot 10^{5}$. Clearly, for these temperatures the previous discussion is inadequate and needs to be modified before we can draw any conclusions about a possible discrepancy between theory and experiment.

The generalization to nonzero temperatures is essentially only useful in the case that the number of particles $N \gg 100$, since then the critical temperature $T_{c}$ is almost equal to the critical temperature

$$
T_{0}=\left(\frac{N}{\zeta(3)}\right)^{1 / 3} \frac{\hbar \omega}{k_{B}} \simeq 0.94 N^{1 / 3} \frac{\hbar \omega}{k_{B}}
$$

of the ideal Bose gas and obeys $k_{B} T_{c} \gg \hbar \omega$ so that we are close to the thermodynamic limit. As a result also the thermal wavelength $\Lambda_{c}=\sqrt{2 \pi \hbar^{2} / m k_{B} T_{c}} \ll \ell$. To understand why this is important, we must realize that at nonzero temperatures the nonlinear Schrödinger equation for the condensate wavefunction is 


$$
i \hbar \frac{\partial}{\partial t} \psi(\vec{x}, t)=\left(-\frac{\hbar^{2}}{2 m} \nabla^{2}+V^{e x t}(\vec{x})+T^{2 B}(\overrightarrow{0}, \overrightarrow{0} ; 0)\left(2 n^{\prime}(\vec{x}, t)+|\psi(\vec{x}, t)|^{2}\right)\right) \psi(\vec{x}, t)
$$

with $n^{\prime}(\vec{x}, t)$ the local density of atoms which are not in the condensate [14, and that the total number of noncondensed particles is of $O\left(\left(k_{B} T / \hbar \omega\right)^{3}\right)$ because $n^{\prime}(\overrightarrow{0}, t)$ is of $O\left(1 / \Lambda^{3}\right)$ and the size of the noncondensed cloud is of $O\left(\ell^{2} / \Lambda\right)$. Therefore, for temperatures that do not obey $k_{B} T \gg \hbar \omega$, the term $2 n^{\prime}(\vec{x}, t)$ in Eq. (29) is negligible compared to $|\psi(\vec{x}, t)|^{2}$. For temperatures $T \gg \hbar \omega / k_{B}$ this does not have to be the case. However, under these conditions we can replace $2 n^{\prime}(\vec{x}, t)$ by a constant since the size of the noncondensed cloud is now much larger than the size of the condensate. Performing then the same analysis as in Sec. IIIA, we find that the potential $V(q)$ is just shifted by a constant.

We thus conclude that for $N \gg 100$ a metastable condensate can exists as long as $N_{0}<N_{m}$, or sufficiently close to the critical temperature. Moreover, the decay rate of the condensate can at all temperatures be calculated by means of the effective action

$$
S[q]=N_{0} \int_{-\hbar \beta / 2}^{\hbar \beta / 2} d \tau\left(\frac{1}{2} m^{*}\left(\frac{d q}{d \tau}\right)^{2}+V(q)\right) .
$$

Hence, for $T \ll \hbar \omega_{0} / k_{B}$ quantum fluctuations dominate and the decay rate is given by Eq. (27) with $N$ replaced by $N_{0}$. For $T \gg \hbar \omega_{0} / k_{B}$, which is the relevant temperature interval if $N \gg N_{m}$, the decay of the condensate is dominated by thermal (or classical) fluctuations and the decay rate is proportional to the Boltzmann factor $e^{-N_{0}\left(V\left(q_{m}\right)-V\left(q_{0}\right)\right) / k_{B} T}$, where $q_{m}$ denotes the position at which $V(q)$ has a maximum. The prefactor of the exponent is always a difficult point, but can be estimated by noting that in the classical limit the action of Eq. (30) implies a probability distribution

$$
P(p, q) \simeq \frac{\beta \omega_{0}}{2 \pi} \exp \left\{-\beta\left(\frac{p^{2}}{2 m^{*} N_{0}}+N_{0}\left(V(q)-V\left(q_{0}\right)\right)\right)\right\}
$$

for the momentum $p$ and the coordinate $q$ of a fictitious classical particle. Using this probability distribution we then easily find from the average flux over the energy barrier that

$$
\Gamma_{0}=\frac{\omega_{0}}{2 \pi} \exp \left\{-\frac{N_{0}}{k_{B} T}\left(V\left(q_{m}\right)-V\left(q_{0}\right)\right)\right\}
$$


Notice that this decay rate is only small if $N_{0}\left(V\left(q_{m}\right)-V\left(q_{0}\right)\right) \ll k_{B} T$. Therefore, a longlived metastable condensate is only possible sufficiently close to the critical temperature, if the total number of atoms obeys $N \ll\left(N_{m}\right)^{3}$.

\section{CONCLUSIONS}

In view of the results obtained above it seems that we have arrived at an explanation of the experiment with atomic ${ }^{7} \mathrm{Li}$, since we have shown that a long-lived metastable condensate is possible if $1 \ll N_{0}<N_{m}$. Unfortunately, this is only partly true because we have not yet considered the mechanical stability of the noncondensed cloud. In particular, we have not shown that the gas is stable (or metastable) at the critical temperature and will, in contrast to what occurs in the homogeneous case [7], not immediately collapse to a dense liquid or solid phase. In our opinion, this is at present the most important question which remains to be answered before we can speak of a theoretical understanding of the Rice experiment.

Finally, we need to discuss the important observation by Dalfovo and Stringari that a condensate with a vortex line through the center of the trap has a value of $N_{m}$ that is significantly larger than $0.23 \ell /|a|$, due to the fact that the local density of the gas must vanish at the point where the phase singularity occurs [15]. In principle, we can also consider the decay of a condensate with a vortex in the framework of the theory presented in Sec. III. We should then first put $\chi(\vec{x}, \tau)=\chi_{v}(\vec{x}, \tau)+\chi^{\prime}(\vec{x}, \tau)$, where $\chi_{v}(\vec{x}, \tau)$ is the phase configuration of a vortex, before we integrate over the nonsingular phase fluctuations $\chi^{\prime}(\vec{x}, \tau)$. The main difficulty, however, will be to find an accurate solution of the coupled dynamical equations for the density profile and the position of the vortex. Although such a calculation would certainly be interesting in its own right, it clearly does not invalidate the main conclusion of our paper that the most important unresolved issue is the stability of the gas without a condensate.

After completion of the work presented in Sec. $\mathrm{IIA}$ of this paper, we received a preprint by Kagan, Shlyapnikov, and Walraven [16] in which these authors also consider the stability 
of an inhomogeneous condensate at $T=0$, and arrive at qualitatively the same results. However, their calculation of the decay rate is completely different from ours and, in particular, does not take phase fluctuations of the condensate into account. As a result, it appears not to correspond to a WKB treatment of the tunneling process.

\section{ACKNOWLEDGMENTS}

I would like to thank Randy Hulet for various enlightening communications and for providing me with a preprint of Ref. [9] that inspired the above. I also thank Keith Burnett and Peter Ruprecht for stimulating discussions on this work at the now historic BEC workshop in Mont Ste Odile. Finally, I am grateful to Michel Bijlsma and Marianne Houbiers for a careful reading of the manuscript. 


\section{REFERENCES}

[1] M.H. Anderson, J.R. Ensher, M.R. Matthews, C.E. Wieman, and E.A. Cornell, Science 269, 198 (1995).

[2] C.C. Bradley, C.A. Sackett, J.J. Tollett, and R.G. Hulet, Phys. Rev. Lett. 75, 1687 (1995).

[3] K.B. Davis, M.-O. Mewes, M.R. Andrews, N.J. van Druten, D.S. Durfee, D.M. Kurn, and W. Ketterle, Phys. Rev. Lett. 75, 3969 (1995).

[4] J.M. Doyle, J.C. Sandberg, I.A. Yu, C.L. Cesar, D. Kleppner, and T.J. Greytak, Phys. Rev. Lett. 67, 603 (1991).

[5] O.J. Luiten, H.G.C. Werij, I.D. Setija, T.W. Hijmans, and J.T.M. Walraven, Phys. Rev. Lett. 70, 544 (1993); and I.D. Setija, H.G.C. Werij, O.J. Luiten, M.W. Reynolds, T.W. Hijmans, and J.T.M. Walraven, Phys. Rev. Lett. 70, 2257 (1993).

[6] E.R.I. Abraham, W.I. McAlexander, C.A. Sackett, and R.G. Hulet, Phys. Rev. Lett. 74, 1315 (1995).

[7] H.T.C. Stoof, Phys. Rev. A 49, 3824 (1994).

[8] R.G. Hulet, (private communication).

[9] P.A. Ruprecht, M.J. Holland, K. Burnett, and M. Edwards, Phys. Rev. A 51, 4704 (1995).

[10] I.H. Duru, H. Kleinert, and N. Ünal, J. Low. Temp. Phys. 42, 137 (1981).

[11] Yu.A. Nepomnyashchii and A.A. Nepomnyashchii, Zh. Eksp. Teor. Fiz. 75, 976 (1978) [Sov. Phys. JETP 48, 493 (1978)].

[12] G. Baym and C. Pethick, Phys. Rev. Lett. 76, 6 (1996), have independently put forward a similar variational approach to calculate the equilibrium shape of the condensate wavefunction. 
[13] S. Coleman, Phys. Rev. D 15, 2929 (1977).

[14] V.V. Goldman, I.F. Silvera, and A.J. Leggett, Phys. Rev. B 24, 2870 (1981).

[15] F. Dalfovo and S. Stringari, (unpublished).

[16] Yu. Kagan, G.V. Shlyapnikov, and J.T.M. Walraven, (unpublished). 